\title{
Design and construction of wireless power transfer system using magnetic resonant coupling
}

\author{
Syed Khalid Rahman ${ }^{1}$, Omar Ahmed ${ }^{2}$, Md. Saiful Islam ${ }^{1}$, A. H. M. Rafiul Awal, \\ Md. Shariful Islam ${ }^{1}$ \\ ${ }^{1}$ Department of Electrical \& Electronic Engineering, World University of Bangladesh, Dhaka, Bangladesh \\ ${ }^{2}$ Department of Electrical \& Electronic Engineering, American International University- Bangladesh, Dhaka, Bangladesh
}

\section{Email address:}

sk.rahman@eee.wub.edu.bd (S. K. Rahman), saifulruetete07@gmail.com (Md. S. Islam), rafi_official@yahoo.com (A. H. M. R. Awal), shariful06eee@gmail.com (Md. S. Islam),omarahmed8923@gmail.com (O. Ahmed)

\section{To cite this article:}

Syed Khalid Rahman, Omar Ahmed, Md. Saiful Islam, A. H. M. Rafiul Awal, Md. Shariful Islam. Design and Construction of Wireless Power Transfer System Using Magnetic Resonant Coupling. American Journal of Electromagnetics and Applications.

Vol. 2, No. 2, 2014, pp. 11-15. doi: 10.11648/j.ajea.20140202.11

\begin{abstract}
Wireless power transmission is the transmission of electrical energy without using any conductor or wire. It is useful to transfer electrical energy to those places where it is hard to transmit energy using conventional wires. In this paper, we designed and implemented a wireless power transfer system using the basics of magnetic resonant coupling. Numerical data are presented for power transfer efficiency of both receivers. Graphs are given to show the comparison of power and efficiency with distance of both receivers.
\end{abstract}

Keywords: Wireless Power Transfer, Magnetic Resonant Coupling, Quality Factor

\section{Introduction}

Wireless power transmission (WPT) is an efficient way for the transmission of electric power from one point to another through vacuum or atmosphere without the use of wire or any substance. By using WPT, power can be transmitted using inductive coupling for short range, resonant induction for mid-range and Electromagnetic wave power transfer. By using this technology, it is possible to supply power to places, which is hard to do using conventional wires. Currently, the use of inductive coupling is in development and research phases.

The most common wireless power transfer technologies are the electromagnetic induction and the microwave power transfer. For efficient midrange power transfer, the wireless power transfer system must satisfy three conditions: (a) high efficiency, (b) large air gap, (c) high power. The microwave power transfer has a low efficiency. For near field power transfer this method may be inefficient, since it involves radiation of electromagnetic waves. Wireless power transfer canbe done via electric field coupling, but electric field coupling provides an inductively loaded electrical dipole that is an open capacitor or dielectric disk. Extraneous objects may provide a relatively strong influence on electric field coupling. Magnetic field coupling may be preferred, since extraneous objects in a magnetic field have the same magnetic properties as empty space.

Electromagnetic induction method has short range. Since magnetic field coupling is a non-radiative power transfer method, it has higher efficiency. However, power transfer range can be increased by applying magnetic coupling with resonance phenomenon applied on. A magnetic field is generated when electric charge moves through space or within an electrical conductor. The geometric shapes of the magnetic flux lines produced by moving charge (electric current) are similar to the shapes of the flux lines in an electrostatic field.

\section{Theory}

Inductive or magnetic coupling works on the principle of electromagnetism. When a wire is proximity to a magnetic field, it induces a magnetic field in that wire. Transferring energy between wires through magnetic fields is inductive coupling. 


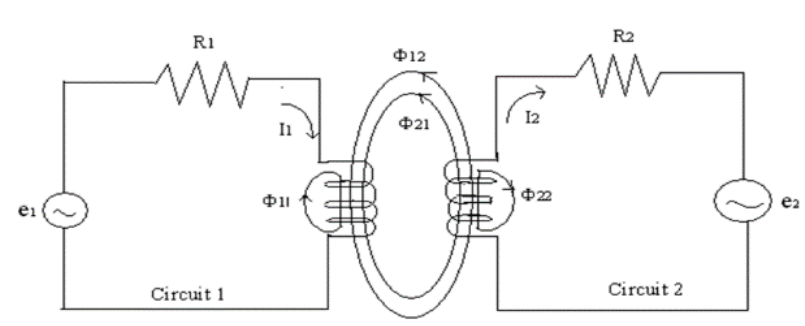

Figure 2.1. Magnetic coupling with four component fluxes

Magnetic resonant coupling uses the same principles as inductive coupling, but it uses resonance to increase the range at which the energy transfer can efficiently take place. Resonance can be two types: (a) series resonance \& (b) parallel resonance. In these both types of resonance, the principle of obtaining maximum energy is same but the methods are quite different.

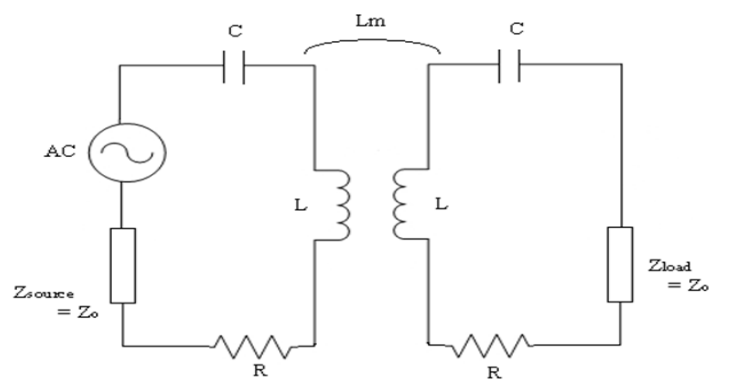

Figure 2.2.Equivalent circuit of Magnetic Resonant Coupling

Quality factor (Q-factor) is a dimensionless parameter that describes the characteristic of an oscillator or resonator, or equivalently, characterizes a resonator's bandwidth relative to its centre frequency. Higher $\mathrm{Q}$ indicates a lower rate of energy loss relative to the stored energy of the oscillator; the oscillations die out more slowly. $Q$ factor determines the qualitative behavior of simple damped oscillators. A system with low quality factor $(Q<1 / 2)$ is said to be overdamped. Such a system does not oscillate at all, but when displaced from its equilibrium steady state output, it returns to it by exponential decay, approaching the steady state value asymptotically. System withhigh quality factor $(Q>1 / 2)$ is said to be underdamped. Underdamped systems combine oscillation at a specific frequency with decay of the amplitude of the signal. A system with an intermediate quality factor $(Q=1 / 2)$ is said to be critically damped. Like an over damped system, the output does not oscillate, and does not overshoot its steady-state output (i.e., it approaches a steady-state asymptote). Like an underdamped response, the output of such a system responds quickly to a unit step input.

The efficiency of the coupled system depends on how much energy is transferred from the transmitted to the receiver circuit.

$$
\eta_{\text {energy }}=\frac{E_{\text {receiver.max }}}{E_{\text {transmitter.max }}}
$$

The maximum energy found on the transmitted $\mathrm{E}_{\text {transmitter.max }}$, is the amount of energy initially put on the input capacitor $\mathrm{C}_{\mathrm{t}}$ by the voltage source $\mathrm{V}_{0}$.

$$
\mathrm{E}_{\text {transmitter.max }} \approx E_{\text {init }}=\frac{1}{2} C_{t} V_{\text {in }}^{2}
$$

Where $V_{\text {in }}$ is the voltage of $C_{t}$ at time $t=0$, during positive pulse duration $\mathrm{C}_{t}$ acts like a voltage source and completes a series loop with the transmitter elements, $\mathrm{R}_{\mathrm{t}}$, $\mathrm{L}_{\mathrm{t}}, \mathrm{C}_{\mathrm{t}}$.

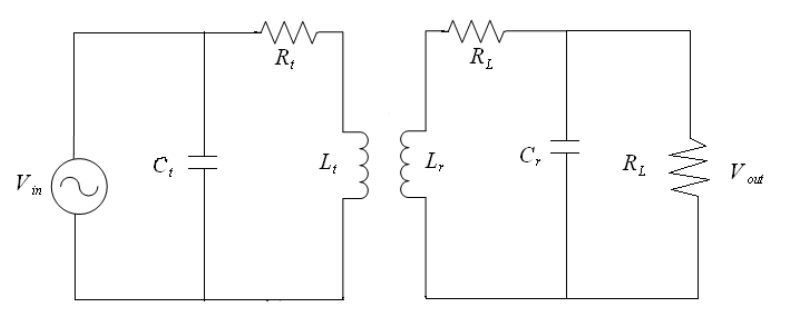

Figure 2.3.Resonant Wireless Power Transmission Circuit Diagram

The maximum energy transferred to the receiver is only a fraction of input energy. The energy found is receiver circuit is:

$$
\mathrm{E}_{\text {receiver }}=\frac{1}{2} \mathrm{~L}_{\mathrm{r}} \mathrm{I}_{\mathrm{r}}^{2}+\frac{1}{2} \mathrm{C}_{\mathrm{r}} \mathrm{V}_{\mathrm{Cr}}^{2}
$$

At maximum voltage level on receiver circuit, current becomes zero and no current flows the circuit. At this point energy stored in receiver inductor is zero because current is zero. Thus, maximized receiver energy is:

$$
\mathrm{E}_{\text {transmitter.max }}=\frac{1}{2} \mathrm{C}_{\mathrm{r}} \mathrm{V}_{\text {out,max }}^{2}
$$

\section{Design}

The following table shows the components which are used to make the oscillator.

Table 3.1.Oscillator Components

\begin{tabular}{ll}
\hline Components Name & Components Value or code \\
\hline Voltage Source, V dc & $15 \mathrm{~V}$ \\
Capacitor, C1 & $100 \mathrm{nF}$ \\
Capacitor, C & $60 \mathrm{nF}$ \\
Resistor, R1 & $100 \mathrm{ohm}$ \\
Resistor, R2 & $100 \mathrm{ohm}$ \\
Resistor, R3 & $10 \mathrm{k} \mathrm{ohm}$ \\
Resistor, R4 & $10 \mathrm{k} \mathrm{ohm}$ \\
Diode, D1 & $1 \mathrm{~N} 4142$ \\
Diode, D2 & $1 \mathrm{~N} 4142$ \\
MOSFET, Q1 & $1 \mathrm{RF} 1010$ \\
MOSFET, Q2 & $1 \mathrm{RF} 1010$ \\
Radio Frequency Choke, L1 & $100 \mathrm{uH}$ \\
Radio Frequency Choke, L2 & $100 \mathrm{uH}$ \\
\hline
\end{tabular}

Our experimental realization of the scheme consists of three coils tuned at the same frequency. An oscillation circuit is connected with a source coil $\mathrm{S}$ is in turn coupled resonant inductively to a load carrying coil $\mathrm{Q}$. The coils are 
made of an electrically conducting copper pipe of a crosssectional radius wound into a helix of single turn, radius r.Then a radio frequency oscillating signal is passed through the coil S, it generates an oscillating magnetic field through the inductance of the coil S, which is tuned at the same frequency by the inductance of the coil and a resonating capacitor $\mathrm{c}$. The load coil $\mathrm{Q}$, tuned at the same resonant frequency receives the power through the magnetic field generated by the source coil S.

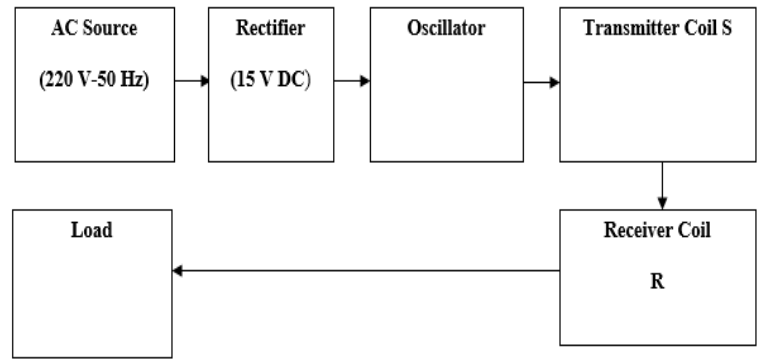

Figure3.1.Block diagram of wireless power transfer system

For the experiment, the source coil and the load coil was constructed using $0.6 \mathrm{~mm}$ copper tube with radius 13 inches. We have constructed two receivers one with a single turn and another with double turn. Transmitter circuit is mainly consists of oscillator circuit. A power supply with rectifier circuit is connected to transmitter circuit with a coil to transmit the power. Receiver circuit includes only the load coil with attached capacitor to receive power.

\section{Transmitter (Source Coil)}

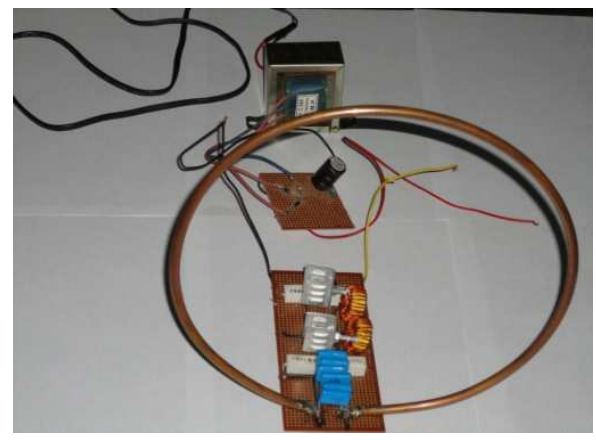

Figure 3.2.Implementation design of transmitter

\section{Receiver 1 (Load Coil 1)}

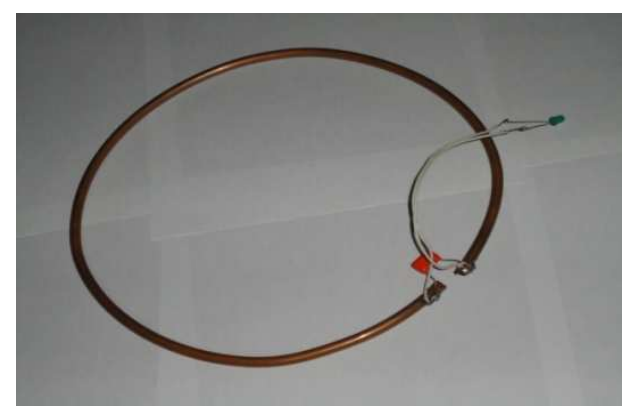

Figure 3.3.Implementation design of receiver 1

\section{Receiver 2 (Load Coil 2)}

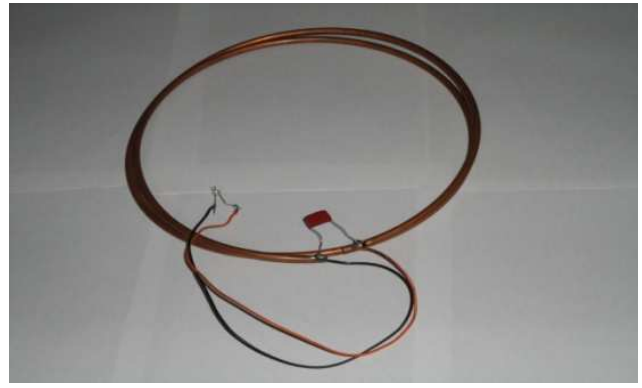

Figure 3.4.Implementation design of receiver 2

Full Project Model with Receiver 1

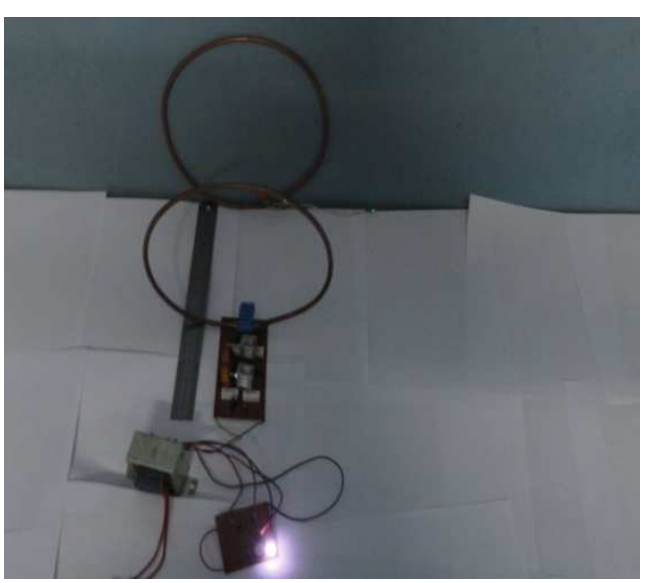

Figure 3.5.Full project model with receiver 1

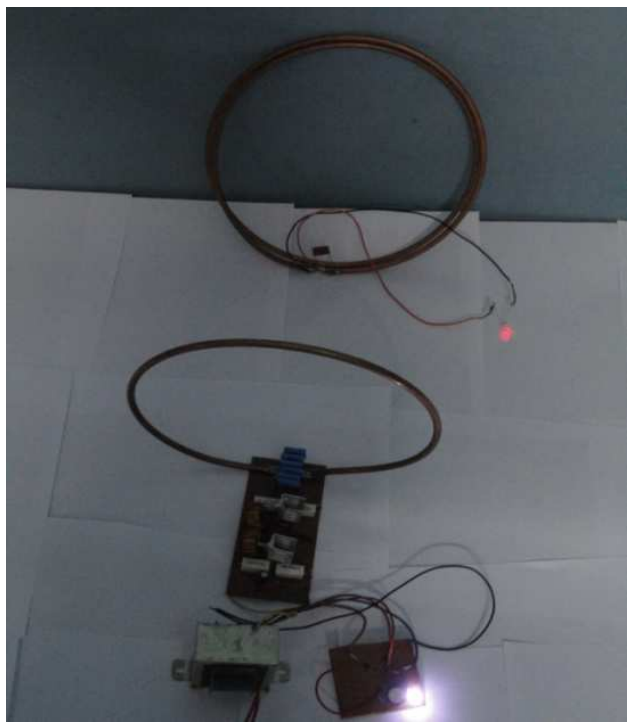

Figure 3.6. Full project model with reciever 2

\section{Calculations}

After completing the basic device we took the measurement of power efficiency of the two receivers. For efficiency calculation, we have taken transmitting and receiving end power of the two receivers respectively. The formula for efficiency calculation is, 


$$
\eta=(\text { Pout } / \text { Pin }) * 100
$$

We used the following formula for power calculation,

$$
\mathrm{P}=\mathrm{VI}
$$

All the tables and Line chart are as follows -

\section{Table for Receiver 1 at Receiving End}

Table 4.1.Power calculation receiving end (receiver 1)

\begin{tabular}{llll}
\hline Distance(cm) & Current(A) & Voltage(V) & Power $(\boldsymbol{\mu W})$ \\
\hline 22 & 0.00024 & 0.037 & 8.88 \\
18 & 0.00062 & 0.049 & 30.38 \\
14 & 0.00095 & 0.082 & 77.9 \\
12 & 0.00143 & 0.108 & 154.44 \\
8 & 0.00211 & 0.169 & 356.59 \\
4 & 0.00448 & 0.252 & 1128.96 \\
\hline
\end{tabular}

Table for Receiver 1 at Sending End

Table 4.2.Power calculation at sending end (receiver 1)

\begin{tabular}{llll}
\hline Distance (cm) & Voltage(V) & Current(A) & Power $(\mu W)$ \\
\hline 22 & 0.15 & 0.00343 & 514.5 \\
18 & 0.162 & 0.00343 & 555.66 \\
14 & 0.186 & 0.00343 & 637.98 \\
12 & 0.209 & 0.00343 & 716.87 \\
8 & 0.244 & 0.00343 & 836.92 \\
4 & 0.346 & 0.00343 & 1186.78 \\
\hline
\end{tabular}

Table for Receiver 2 at Receiving End

Table 4.3.Power calculation at receiving end (receiver 2)

\begin{tabular}{llll}
\hline Distance(cm) & Current(A) & Voltage(V) & Power $(\boldsymbol{\mu W})$ \\
\hline 40 & 0.00005 & 0.0001 & 0.005 \\
35 & 0.00009 & 0.0002 & 0.018 \\
30 & 0.00015 & 0.0005 & 0.075 \\
25 & 0.00031 & 0.0006 & 0.186 \\
20 & 0.00069 & 0.0106 & 7.314 \\
15 & 0.00199 & 0.0253 & 50.347 \\
10 & 0.00259 & 0.0528 & 136.752 \\
5 & 0.00705 & 0.119 & 838.95 \\
\hline
\end{tabular}

Table for Receiver 2 at Sending End

Table 4.4.Power calculation at sending end (receiver 2)

\begin{tabular}{llll}
\hline Distance (cm) & Voltage(v) & Current(A) & Power $(\boldsymbol{\mu W})$ \\
\hline 40 & 0.207 & 0.00343 & 710.01 \\
35 & 0.283 & 0.00343 & 970.69 \\
30 & 0.358 & 0.00343 & 1227.94 \\
25 & 0.387 & 0.00343 & 1327.41 \\
20 & 0.4 & 0.00343 & 1372 \\
15 & 0.435 & 0.00343 & 1492.05 \\
10 & 0.487 & 0.00343 & 1670.41 \\
5 & 0.509 & 0.00343 & 1745.87 \\
\hline
\end{tabular}

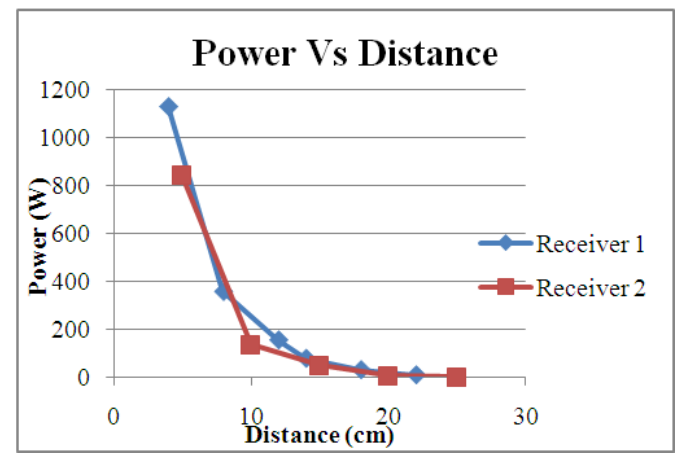

Figure 4.1.Power Vs Distance chart for both receivers at receiver end

Table for Efficiency Calculation for Receiver 1

Table 4.5.Efficiency calculation for receiver 1

\begin{tabular}{llll}
\hline Distance & $\begin{array}{l}\text { Sending end } \\
\text { power (Pin) }\end{array}$ & $\begin{array}{l}\text { Receiving end } \\
\text { power (Pout) }\end{array}$ & $\begin{array}{l}\text { Efficiency } \\
\boldsymbol{\eta} \text { (receiver 1) }\end{array}$ \\
\hline 22 & 514.5 & 8.88 & 1.725948 \\
18 & 555.66 & 30.38 & 5.467372 \\
14 & 637.98 & 77.9 & 12.21041 \\
12 & 716.87 & 154.44 & 21.54366 \\
8 & 836.92 & 356.59 & 42.60742 \\
4 & 1186.78 & 1128.96 & 95.12799 \\
\hline
\end{tabular}

Table for Efficiency Calculation for Receiver 2

Table 4.6.Efficiency calculation for receiver 2

\begin{tabular}{llll}
\hline Distance & $\begin{array}{l}\text { Sending end } \\
\text { power (Pin) }\end{array}$ & $\begin{array}{l}\text { Receiving end } \\
\text { power (Pout) }\end{array}$ & $\begin{array}{l}\text { Efficiency } \\
\boldsymbol{\eta} \text { (receiver 2) }\end{array}$ \\
\hline 40 & 710.01 & 0.005 & 0.000704 \\
35 & 970.69 & 0.018 & 0.001854 \\
30 & 1227.94 & 0.075 & 0.006108 \\
25 & 1327.41 & 0.186 & 0.014012 \\
20 & 1372 & 7.314 & 0.53309 \\
15 & 1492.05 & 50.347 & 3.374351 \\
10 & 1670.41 & 136.752 & 8.186733 \\
5 & 1745.87 & 838.95 & 48.05341 \\
\hline
\end{tabular}

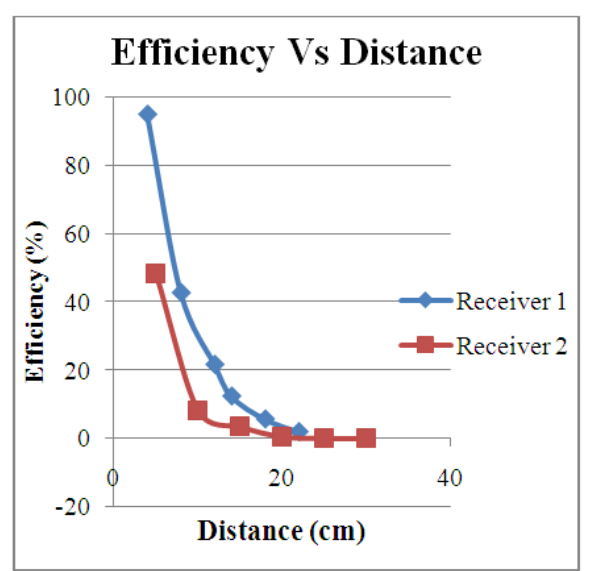

Figure 4.2. Efficiency Vs Distance chart for both receivers 


\section{Discussion}

The first idea was that in the circuit Vacuum tube transistors would be used which provides much higher power than the typical power MOSFETs. Later this idea was eliminated, as vacuum tube transistor could not be found in the shops.

In the local market low equivalent series resistance, (ESR) capacitors are not available. For the oscillator circuit presented, low ESR polypropylene capacitors are highly recommended to handle the high current flowing through the LC tank. Moreover, other type of capacitor creates high spikes in the sinusoidal wave at the LC tank circuit and affects the MOSFETs. However, Mylar capacitors at first were used which has polyester as the dielectric. The circuit became unstable by using this type of capacitor. Later MKP capacitors were used which performed much better.

Toroid cores used to construct the radio frequency chokes are also not available in the local market. They were collected from two old computer power supplies. Experiments were done using various high-speed n-channel MOSFET. MOSFETs with low drain to source on resistance and higher power dissipation found to perform better in the circuit.

At first, the transmitter circuit did not oscillate; instead one MOSFET and inductor heated up rapidly. Later it was found that, short circuit was caused by the voltage of power supply rising too slowly on power-up. This was solved by using a switch on the low voltage side that is immediately between the oscillator circuit and the rectifier.

After the oscillator circuit started to oscillate very little power was available on the load coil. Because the receiver coil was slightly out of resonance, it could not pick up the power properly. This was solved by building both LC-tank circuits with identical loops and capacitances, so that both the circuits have the same resonant frequency.

\section{Conclusion}

The goal of this project was to design and implement a wireless power transfer system via magnetic resonant coupling. After analyzing the whole system systematically for optimization, a system was designed and implemented. Experimental results showed that significant improvements in terms of power-transfer efficiency have been achieved. Measured results are in good agreement with the theoretical models.

We have described and demonstrated that magnetic resonant coupling can be used to deliver power wirelessly from a source coil to a with a load coil with an intermediate coil placed between the source and load coil and with capacitors at the coil terminals providing a sample means to match resonant frequencies for the coils. This mechanism is a potentially robust means for delivering wireless power to a receiver from a source coil.

\section{References}

[1] Zia A. Yamayee and Juan L. Bala, Jr., "Electromechanical Energy Devices and Power Systems", John Wiley and Sons, 1947, p. 78

[2] Simon Ramo, John R. Whinnery andTheodore Van Duzer, "Fields and Waves in Communication Electronics", John Wiley \& Sons, Inc.; 3rd edition (February 9, 1994)

[3] S. Kopparthi, Pratul K. Ajmera, "Power delivery for remotely located Microsystems," Proc. of IEEE Region 5, 2004 Annual Tech. Conference, 2004 April 2, pp. 31-39.

[4] Tomohiro Yamada, Hirotaka Sugawara, Kenichi Okada, Kazuya Masu, andAkio Oki, "Battery-less Wireless Communication System through Human Body for invivo Healthcare Chip,"IEEE Topical Meeting on Silicon Monolithic Integrated Circuits in RF Systems"

[5] "Category:Radio spectrum -Wikipedia, the free encyclopedia," [online document], 2004 Aug 26 [cited 12/11/04], http://en.wikipedia.org/wiki/Category:Radio_spectrum. 